\title{
The Spatial-Temporal Distribution of the Coupling between Urbanization and Farmland Resources in the Mainland of China
}

\author{
Xiang Gao*, Lei Cao, Xiaohua Ma, Hao Zhao \\ College of Earth and Environmental Sciences, Lanzhou University, Lanzhou, P. R. China
}

Email address:

xgao@1zu.edu.cn (Xiang Gao)

${ }^{*}$ Corresponding author

\section{To cite this article:}

Xiang Gao, Lei Cao, Xiaohua Ma, Hao Zhao. The Spatial-Temporal Distribution of the Coupling between Urbanization and Farmland Resources in the Mainland of China. Journal of Energy and Natural Resources. Vol. 5, No. 5, 2016, pp. 59-66. doi: $10.11648 /$ j.jenr.20160505.12

Received: September 20, 2016; Accepted: October 2, 2016; Published: October 31, 2016

\begin{abstract}
An evaluation index system was constructed for the coupling between urbanization and farmland resources, based on data from 31 provinces in the mainland of China. Using the Grey Relational Analysis method, the spatial-temporal variations of the coupling between them can be revealed. The conclusions of this study were: (1) the stress of urbanization on farmland resources is dominated by economic urbanization, while this kind of constraint also produces the strongest influence on the status (endowment) of the arable resources; (2) viewed over time, the coupling degree in Chinese provinces between urbanization and farmland resources shows volatility and internal differences: East $>$ West $>$ Central regions, and the causes for these differences are also discussed; (3) The interaction between them is spatially strong, and the coupling degree is relatively close and high, showing the coexisting features of complexity and specificity. Combining the coupling with the urban level the 31 provinces can be categorized into four types: Low-level Coupled Mode, Antagonism Mode, Transition Mode and Coordination Mode.
\end{abstract}

Keywords: Urbanization, Farmland Resources, Coupling Degree, Grey Relational Analysis, China

\section{Introduction}

Urbanization is the inevitable result of modernization, especially for the developing countries. The 2012 Blue Book of Chinese Society, published by the Chinese Academy of Social Sciences, stated that in 2011, for the first time in thousands of years, China's urban population surpassed $50 \%$ of the total population. This milestone in China's development history indicates that China has entered into a new growth phase focused on urban society. Urbanization has now become a gigantic engine pushing the social and economic development of China, following on the earlier phases of industrialization. From a geographical perspective, urbanization is the process whereby regional space expands constantly, and the urban landscape evolves ceaselessly. It is therefore unavoidable that some types of lands, most notably cultivated lands, have been transformed into residential, commercial \& public land, during urbanization. There is, however, an inherent contradiction between urbanization and farmland resource protection. Arable land, the most basic non-renewable natural resource, is essential to human life, and keeping a certain quantity and quality of arable land is key to sustainable development [1]. In the past 30 years, Chinese farmland resources have steadily decreased. Economic and spatial urbanization have led to an increasing takeover of farmland by urban development and to the conflict between "eat versus build." Farmland protection is increasingly critical [2]. In 2011, land devoted to new urban construction in China came to 0.4467 million hectares, compared to 0.4267 million hectares in 2010, an annual increase of about $4.7 \%[3]$. Undoubtedly, the next decade or so will be a critical period for the development of Chinese urbanization as well as a time of increasing land-use conflicts. Therefore, research on the relationship between urbanization and farmland resources has become a major focus for geographers and land-use planners, and achieving a balance between these two land uses is essential to continued sustainable development. 
It has been traditionally assumed that increased urbanization inevitably leads to dwindling plantation acreage and decreased quality of the remaining arable land. According to regression analysis on urbanization and the total cultivated area of Jiangsu province from 1978 to 1997 , performed by $\mathrm{Yu}$ [4], the amount of arable land decreases by $13,688.3$ hectares with every $1 \%$ increase in the level of urbanization. After researching the relationship between urbanization and farmland in Chengdu city from 1978 to 2007, Chen et al. [5] concluded that urbanization increase and farmland acreage decrease showed a significant degree of correlation. Tan et al. [6] and Zhang [7] also carried out research on this correlation and proposed three explanations: (a) The real-estate and development "crazes" resulted in the conversion of excessive amounts of farmland; (b) Large cities have expanded horizontally rather than vertically, resulting in a "pancake" type of development, often absorbing nearby small towns; and (c) Central governmental control over land use is weak in some regions. Although government theoretically has the first claim on available land, in practice this authority is often abdicated, resulting in "multiple approval and multiple control" as various private entities vie for land development. While it is undeniable that urban development brings with it a series of farmland problems (e.g., loss of farmland, lowered quality of farmland), this viewpoint considers only the conversion of farmland to urban use caused by urban population increase, ignoring the space saved by more intensive utilization of land. Therefore, many scholars doubt the traditional viewpoints enumerated above and suggest there is a "Kuznets curve" relationship between urbanization level and farmland loss [8]. That is, farmland loss shows a reversed " $U$ " shape relationship with urban development, first rising and then falling. The proposed response to this phenomenon is an urbanization strategy of developing large and mid-sized cities while limiting the horizontal expansion of small towns [9][10], meanwhile enhancing the intensity of land use [11].

Currently, studies on the relationship between urbanization and farmland loss, both at home and abroad, use the method of "problem statement - causal mechanism regulations and standards - scenario prediction - response strategies" [12] [13]. Yet research on the feedback loop between urban development and farmland variation has been insufficient, adding little to the understanding of how these two trends interact. To address this deficit, this study adopted Grey Relational Analysis (GRA), using secondary data from 31 Chinese provinces for the years 1990 to 2008. The purpose was to assess the coupling mechanism between urbanization and farmland, on both a temporal and a spatial basis, and to propose countermeasures and suggestions for the coordinated development of these two resources.

\section{Methodology}

\subsection{Indicators}

The interactions between urbanization and farmland resources are so complex that a single indicator cannot truly reflect the internal mechanism and laws between the two uses. Therefore, while referencing previous studies [14] and adhering to principles of scientific research (i.e., comprehensiveness, universality, and availability), this study adopted an analysis approach, using theoretical and expert consultation to develop a common measurement index. To arrive at this base index, the correlation coefficient method and principal component analysis were applied to screen the general assessment indicator system, in an effort to maximize information included in the correlation coefficients, while minimizing overlap information.

Urbanization is a complex system involving interactions among population, society, and economy. Population growth, economic development, social progress, and spatial expansion are interrelated and build on each other [15]. Among these four elements, economic development represents the foundation; population increase and geographic expansion are the manifestations of urbanization; and social progress is the ultimate result or goal. Therefore, using as a foundation the urban integrated measurement index system confirmed by Chen et al. [1], from the four aspects-population, economy, society, and spatial expansion-we sought the maximum number of features to create a comprehensive pool of urbanization features, choosing 18 items to form an index of urbanization. Because the index centers on the human use of farmland resources, it reveals the impacts of human activities.

As one of the basic supports for urban development, farmland resources are both the endogenous variable and the important external force for urbanization and socioeconomic development. The indicator system of farmland resources can therefore be described by the quantity descriptor, which reflects the status and changes of the farmland resources affected by human activity. This study, based on Press-State-Response (PSR) from three aspects of farmland resource-stress, status and socioeconomic response-describes the farmland resource system [16]. Twelve items were selected to form the index system of the farmland resources (Table 1).

Table 1. Indicators for Urban Systems and Farmland Resource Systems.

\begin{tabular}{ll}
\hline System & Index \\
\hline & $\mathrm{X}_{1}$ : Proportion of urban population \\
& $\mathrm{X}_{2}$ : Secondary industry employment proportion \\
& $\mathrm{X}_{3}$ : Tertiary industry employment proportion \\
& $\mathrm{X}_{4}$ : Built-up area population density \\
& $\mathrm{X}_{5}$ : Per Capita GDP \\
& $\mathrm{X}_{6}$ : Per Capita Total Industrial Output Value \\
\hline
\end{tabular}




\begin{tabular}{ll}
\hline System & Index \\
\hline & $\mathrm{X}_{7}:$ Proportion of Output Value of Tertiary Industry \\
$\mathrm{X}_{8}:$ Fixed assets investment per capita \\
$\mathrm{X}_{9}:$ Average wage for on-post staff \\
$\mathrm{X}_{10}:$ Per capita disposable income for urban dwellers \\
$\mathrm{X}_{11}$ : University students for every ten thousand people \\
$\mathrm{X}_{12}:$ Doctors for every ten thousand people \\
$\mathrm{X}_{13}:$ Technicians for every ten thousand people \\
$\mathrm{X}_{14}:$ Per capita public green areas \\
$\mathrm{X}_{15}:$ Ratio of built-up areas \\
$\mathrm{X}_{16}:$ Per capita area of built-up areas \\
$\mathrm{X}_{17}:$ Per capita road area \\
$\mathrm{X}_{18}:$ Town density \\
$\mathrm{Y}_{1}:$ Population density \\
$\mathrm{Y}_{2}:$ GDP density \\
$\mathrm{Y}_{3}:$ Unit area water resources quantity \\
$\mathrm{Y}_{4}:$ Unit area application quantity of chemical fertilizer \\
$\mathrm{Y}_{5}:$ Per capita farmland \\
$\mathrm{Y}_{6}:$ Ratio of effective irrigation area \\
$\mathrm{Y}_{7}:$ Productivity of arable land \\
$\mathrm{Y}_{8}:$ Distribution coefficient of land income \\
$\mathrm{Y}_{9}:$ Per-hectare mechanical power \\
$\mathrm{Y}_{10}:$ Per-hectare output \\
$\mathrm{Y}_{11}:$ Entropy of industrial structure \\
$\mathrm{Y}_{12}:$ Discharge rate of Industrial wastewater \\
Farmland
\end{tabular}

\subsection{Data Sources and Preprocessing}

Thirty-one provinces and autonomous regions in the mainland of China comprised the basic units of research. The main data sources included the Statistical Yearbook (2009) for each province, the China Statistical Yearbook (1990-2009), the Statistical Bulletin for National Economic and Social Development (2009), and the China Agricultural Statistics Yearbook (2009). In order to consider the differences among the original index data dimensions and more conveniently calculate crosswise comparison among the 31 provinces, before GRA analysis, the range and standard deviation were calculated to reduce the variable to one dimension [17]:

$$
Z_{i j}=\left(Z_{i j}-\min _{i} Z_{i j}\right) /\left(\max _{i} Z_{i j}-\min _{i} Z_{i j}\right)
$$

Where $Z_{i j}$ is the attribute value of a given index,

$\max _{i} Z_{i j}, \min _{i} Z_{i j}$ are the maximum and minimum values of a target.

\subsection{Methods}

Grey Relational Analysis (GRA) supplies a quantized measurement for examining trends in system development, which is appropriate for dynamic analysis [18]. To reveal the spatial-temporal differentiation of coupling between Chinese urbanization and farmland resources requires relating degree mode and coupling mode to form the primary measurement, as seen by Liu and Li [19]. The measurement starts with the GRA degree. Its correlation coefficient is: $\xi_{i}(j)(t)=\frac{\min _{i} \min _{j}\left|Z_{i}{ }^{X}(t)-Z_{j}^{Y}(t)\right|+\rho \cdot \max _{i} \max _{j}\left|Z_{i}{ }^{X}(t)-Z_{j}^{Y}(t)\right|}{\left|Z_{i}{ }^{X}(t)-Z_{j}^{Y}(t)\right|+\rho \cdot \max _{i} \max _{j}\left|Z_{i}^{X}(t)-Z_{j}^{Y}(t)\right|}$

Where $Z_{i}{ }^{X}(t)$ and $Z_{j}{ }^{Y}(t)$ is the standard value of the indexes of the farmland resources system and the urbanization system in each session, respectively; $\rho$ is the resolution ratio, which is generally 0.5 ; and $\xi_{i}(j)(t)$ is the correlation coefficient of $t$ moment.

According to the sample $\mathrm{K}$, the average correlation coefficient and an mxl degree of association matrix $\gamma_{\text {can be }}$ calculated, reflecting the complex coupling relationship between urbanization and farmland resources. ( $\mathrm{K}$ can use a time series to reveal the sequential variation in the variables, and, with cross-section data, calculate the spatial interaction among the variables). Through the comparison of the $\gamma_{i j}$ dimensions of each degree of association, it can be shown that some factors in the farmland resources have close relationships with urbanization development, while some other factors have few impacts on the role of urbanization. If $\gamma_{i j}=1$, then a given index $Z_{j}{ }^{Y}(t)$ of farmland resources is highly correlated with a corresponding index $Z_{i}{ }^{X}(t)$ of urbanization, and states $Z_{i}{ }^{X}(t)$ and $Z_{j}{ }^{Y}(t)$ have the same variation rules. If $\gamma_{i j}$ falls between 0 and 1: If the value of $\gamma_{i j}$ is larger, the relevance is larger, and accordingly the coupling is stronger, and vice versa. When the index is $0<\gamma_{i j} \leq 0.35$, the degree of association, and thereby the coupling effect between the two systems, is weak. When the index is $0.35<\gamma_{i j} \leq 0.65$, the degree of association, and thereby the coupling effect, is moderate. When the index is $0.65<\gamma_{i j} \leq 0.85$, the degree of association, and the coupling effect, is relatively strong. When the index is $0.85<\gamma_{i j} \leq 1$, the degree of association, and the coupling effect, is very strong.

Next, the average value by rows or columns was determined by the correlation calculation, resulting in the average correlation degree shown in Equation (3). The main coercion factors were chosen according to the size and corresponding range of the value, such as the stressing factors of the urbanization on farmland resources and the restraining factors of plantation resources with urbanization.

$$
\left\{\begin{array}{l}
d_{i}=\frac{1}{l} \sum_{j=1}^{l} \gamma_{i j}(i=1,2, \cdots \cdots, m ; j=1,2, \cdots \cdots, l) \\
d_{j}=\frac{1}{m} \sum_{i=1}^{l} \gamma_{i j}(i=1,2, \cdots \cdots, m ; j=1,2, \cdots \cdots, l)
\end{array}\right.
$$

where $\gamma_{i j}$ is the degree of association; $l$ and $m$ are the index numbers of the urbanization system and the farmland resources system, respectively.

In order to judge the overall coupling degree between urbanization and farmland resources using Equation (1), a coupling degree mode was built between urbanization and farmland. The strength of coupling between the two elements can be evaluated quantitatively from the angle of space-time 
through this mode.

$$
\mathrm{C}(t)=\frac{1}{m \times l} \sum_{i=1}^{m} \sum_{j=1}^{l} \xi_{j}(t)
$$

where $\mathrm{C}(t)$ is the coupling degree of both systems.

The coupling degree has been used previously to refer to the degree of link tightness in the field of computers; however, in the relational analysis of urbanization and farmland resources, coupling degree refers to both their relation tightness degree and their interaction degree. Thus, its physical significance is, if the coupling degree is higher, the relation between urbanization and farmland resources is tighter, the mutual effects are increased, and the conditionality is strong and vice versa.

\section{Results and Discussion}

\subsection{The Coupling Features Between Chinese Urbanization and Farmland Resources}

In 2009, the calculated correlation degree between Chinese urbanization and farmland resources was above 0.5 , a medium effect, showing a strong connection between the two systems. The coupling details between the two elements in terms of the main stressing factors of urbanization on the farmland resources (Table 2) and the main restraining factors of the farmland resources with urbanization (Table 3 ) can be made clear based on Equation (3).

\subsection{Characteristics of Urbanization Stress on Cultivated Land}

The stress of urbanization on arable land is a direct result of the expansion and propulsion of the urban area, the pattern of change, and the extension of landscape. In the process of the urbanization stress, all kinds of economic activities (e.g., productive activities of urban residents, production and marketing of enterprises, traffic, utilization of resources, and energy consumption) act upon regional farmland resources. Thus, the stress on cultivated land from economic urbanization is exacerbated. Table 2 shows the correlation degree between each subsystem of urbanization and farmland resources, from large to small: economic urbanization $(0.693)>$ social urbanization $(0.681)>$ population urbanization $(0.678)>$ spatial urbanization (0.655). At the same time, all of the association degrees of the four kinds of urbanization with farmland resources are greater than 0.65 , indicating that the stress of urbanization on farmland resources can be classified in the stronger stress category. The development of Chinese urbanization has eliminated the top-down government-led model since the reform and opening-up to the outside world, but now development is dominated by industrialization with notable urbanization level and distinct acceleration. In its early stages, gains in urbanization and industrialization resulted only in increased resource consumption and environmental pollution. Now, however, with the different development pattern between urbanization and industrialization, a more serious consequence is that regulations protecting farmland from urbanization have not kept up with the demand for development land, and farmland depletion is out of control. China's economic development is driving urban development along the route of "pollute first, treat later." Therefore, it has become essential to determine the urbanization stress on farmland resources in the mainland of China.

Table 2. Stress Factor Matrix between Urbanization and Farmland Resources.

\begin{tabular}{|c|c|c|c|c|c|c|c|c|c|}
\hline & \multicolumn{9}{|c|}{ Urbanization } \\
\hline \multirow{6}{*}{$\begin{array}{l}\text { Farmland } \\
\text { Resources }\end{array}$} & \multicolumn{4}{|c|}{ Population Urbanization (0.678) } & \multicolumn{5}{|c|}{ Economic Urbanization (0.693) } \\
\hline & $\mathrm{X}_{1}$ & $\mathrm{X}_{2}$ & $\mathrm{X}_{3}$ & $\mathrm{X}_{4}$ & $\mathrm{X}_{5}$ & $\mathrm{X}_{6}$ & $\mathrm{X}_{7}$ & $\mathrm{X}_{8}$ & $\mathrm{X}_{9}$ \\
\hline & 0.703 & 0.662 & 0.708 & 0.638 & 0.711 & 0.729 & 0.667 & 0.735 & 0.624 \\
\hline & \multicolumn{4}{|c|}{ Social Urbanization $(0.681)$} & \multicolumn{5}{|c|}{ Spatial Urbanization (0.655) } \\
\hline & $\mathrm{X}_{10}$ & $\mathrm{X}_{11}$ & $\mathrm{X}_{12}$ & $\mathrm{X}_{13}$ & $\mathrm{X}_{14}$ & $\mathrm{X}_{15}$ & $\mathrm{X}_{16}$ & $\mathrm{X}_{17}$ & $\mathrm{X}_{18}$ \\
\hline & 0.616 & 0.706 & 0.637 & 0.735 & 0.709 & 0.691 & 0.630 & 0.631 & 0.666 \\
\hline
\end{tabular}

Among all indicators of urbanization, the top five that threaten farmland resources are (a) per capita fixed assets investment (0.735), (b) number of science and technology personnel per 10,000 people $(0.735)$, (c) per capita GDP (0.729), (d) per capita gross industrial output value (0.711), and (e) per capita public green areas (0.709). Urbanization's stress on farmland resources is mainly reflected in economic and social structures. The research by Chen et al.[1] on each subsystem of urbanization showed that rapid industrial development is the main factor in the evolution of economic urbanization. In the early stages of urbanization, China showed a typical pattern of urban expansion and extensive land development, with little thought to efficient land use. In some cities, where the concepts of urban development were not applied, suburban sprawl dominated. Some medium-sized and small towns, which had weak governmental regulations and limited economic resources, raced to lower land prices and designate development zones for attracting investment, resulting in large areas of inefficient land use and loss of this valuable national asset. It is thus clear that the pattern of extensive land use in early urban development went against the intensive utilization of land, and put a heavy stress on farmland resources.

\subsection{Characteristics of the Restrictive Effect of Farmland on Urbanization}

The restrictive effect of farmland on urbanization is due to a process of interactions between urbanization and land resources. With the advance of urbanization, urban land scale, 
structure, and efficiency are likely to change. Or, from the farmland perspective, farmland shortage, reduced quality, and unbalanced distribution will restrain urbanization. From Table 3 , it can be seen that the restraint of farmland resources upon urbanization is strongest $(0.687)$, followed by cultivated land resource pressure (0.683), and relatively weak economic response (0.663). In total, all three reflect a strong constraint. Urbanization itself is an approach of intensively using land, mitigating the pressure on farmland by transferring rural populations to urban built-up areas. According to the Chinese Statistical Yearbook, for 30 years, from 1978 to 2008, the Chinese urbanization level rose to $45.68 \%$ from $17.92 \%$, and the urban construction area expanded from $8,842 \mathrm{~km}^{2}$ to $36,295 \mathrm{~km}^{2}$. In other words, the annual urbanization level increased by almost $1 \%$, while the urban built area expanded by $989 \mathrm{~km}^{2}(10.35 \%)$, far higher than in other countries. Especially notable is that, despite leading the world in construction per capita, China has made limited contributions to urban improvement. It is thus clear that the excessive scale and expansion of urban land use will lead not only to an extremely low intensity of urban land use, but also to the urban development of a large amount of land. This situation will create a shortage of farmland, lower farmland quality, and to some degree restrict the process of Chinese urbanization.

From the index level, the constraining effect of farmland resources on urbanization belongs in the category of intermediate strong effect (the degree of association is 0.35 to $0.85)$. The five highest indicators of urbanization restriction are (a) farmland productivity $(0.732)$, (b) population density $(0.727)$, (c) the attainment rate of industrial waste water discharge (0.724), (d) per capita farmland area (0.699), (e) and information entropy of industrial structure (0.693). These indicators reflect limited farmland, an unsustainable industrial structure, and serious pollution-evidence of an obvious restricting effect of the farmland resources on urbanization. China is a huge agricultural country, feeding about $22 \%$ of the world population using only $7 \%$ of the world's arable land. The relative lack of per capita farmland is a fundamental reality in China. Thus, it can be seen that the inefficiency and the unreasonable development and utilization of farmland resources are the key factors restricting the development of Chinese urbanization.

Table 3. Factors Matrix of Constraint of Chinese Farmland Quality on Urbanization.

\begin{tabular}{|c|c|c|c|c|c|c|c|c|c|c|c|c|}
\hline \multirow{4}{*}{ Urbanization } & \multicolumn{12}{|c|}{ Farmland Resources } \\
\hline & \multicolumn{4}{|c|}{$\begin{array}{l}\text { Pressure of Farmland Resources } \\
(\mathbf{0 . 6 8 3 )}\end{array}$} & \multicolumn{4}{|c|}{$\begin{array}{l}\text { Condition of Farmland Resources } \\
(0.687)\end{array}$} & \multicolumn{4}{|c|}{ Socioeconomic Response (0.663) } \\
\hline & $Y_{1}$ & $\mathbf{Y}_{2}$ & $\mathbf{Y}_{3}$ & $\mathbf{Y}_{4}$ & $Y_{5}$ & $Y_{6}$ & $\mathbf{Y}_{7}$ & $\mathbf{Y}_{8}$ & $\mathbf{Y}_{9}$ & $Y_{10}$ & $Y_{11}$ & $Y_{12}$ \\
\hline & 0.727 & 0.667 & 0.681 & 0.657 & 0.699 & 0.662 & 0.732 & 0.655 & 0.686 & 0.550 & 0.693 & 0.724 \\
\hline
\end{tabular}

The sequential variation of coupling between urbanization and farmland resources The coupling between urbanization and farmland resources is expressed not only by the interaction complexity of respective factors, but also by time sequence and spatial diversity. To reveal the temporal-spatial variation of the coupling between Chinese urbanization and farmland resources, the sample points of every provincial calendar year for cross section data were calculated using Equation (4). The time-series changes of the coupling of urbanization and farmland resources from 1990-2008 are shown for China as a whole, as well as for the eastern, central, and western regions of China. Figure 1 summarizes the characteristics of temporal coupling changes.

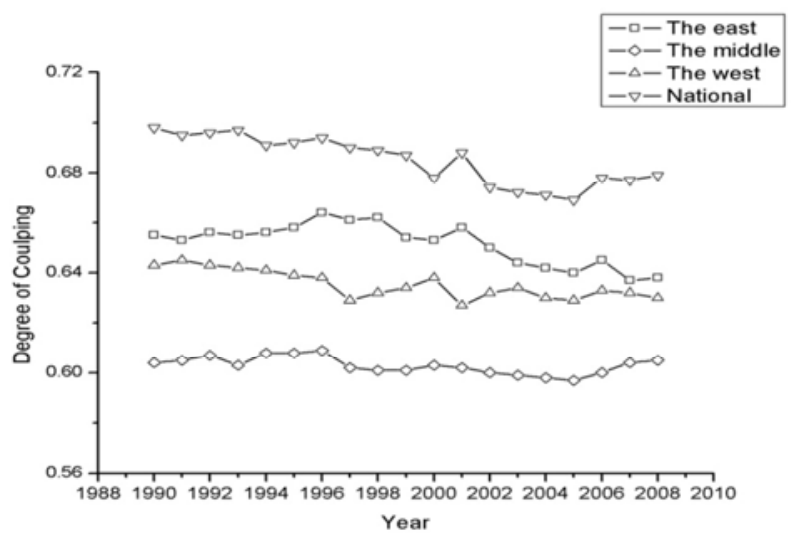

Figure 1. The coupling degree shows a fluctuating downward trend with an obvious overall volatility.
In 1996, Chinese urbanization reached $30.68 \%$ (the proportion of urban population). According to international experts, the development of Chinese urbanization has entered into the phase of rapid development (urban population proportion $>30 \%$ ). Urbanization development needs the support of funding, resources, and population transfer; therefore, the urbanization and farmland resources should show a state of constant adjustment between the modes of antagonism and coordination, and the trend lines in Figure 1 support this view. From 1990 to 2008, the coupling degree of urbanization and farmland resources fluctuated between 0.665 and 0.635 , peaking in 1996 at approximately 0.695 and then declining after 2005. The changes could be divided into two stages with the year 1996 as the boundary. The period from 1990 to 1996 was the secular change stage, when change throughout the entire nation and across regions appeared to be mild, while the change from 1996 to 2008 was acute. From 1989 to 2000, due to the policy of strictly controlling the scale of cities and allowing reasonable development of medium-sized and small cities, the comprehensive construction of an affluent society was the goal, with full prosperity of the rural economy and acceleration of the urbanization process. The urbanization strategy adhered to the coordinated development of large, medium, and small cities and small towns. Urbanization promoted the development of intensive utilization of farmland resources. Thus, the intensity of the interaction between urbanization and farmland resources was abated, and their coupling degree was on the decline.

There are obvious differences in the fluctuations of the coupling degree between urbanization and farmland resources 
among eastern, central, and western China. Overall, the coupling degree increased in the east, then the central, and then the west, in turn. Specifically, the coupling degree of the eastern region increased slowly before 1996, fluctuated between 1996 and 2001, and then significantly declined after 2001. The coupling degree of the central region gradually changed by approximately 0.6 between 1990 and 2008. The coupling degree of the western region slowly decreased before 1996, and then fluctuated between 1996 and 2008. These results demonstrate the complex changes of the coupling degree, which were due mainly to unbalanced development strategies since Chinese reform and opening up to the outside world. Under the unbalanced development strategy, the amount of construction land in the eastern region is much greater than in the central or western regions. In addition, non-farm configuration of farmland resources and other factors of production, both spatial and temporal, have been concentrated in the eastern coastal region. Rapid industrialization and urbanization have transformed that area into the most economically developed region of China, while resource utilization and economic development in the mid-west region have lagged behind those in the east. Consequently, the coupling degree of urbanization and farmland resources in the mid-west region is less than that of the eastern region.

\subsection{Spatial Differences in the Coupling Between Urbanization and Cultivated Land}

Using cross-section data from 31 provinces from 1990 to 2008, this study applied Equation 4 to find out the degree of coupling between Chinese urbanization and farmland resources (Figure 2). The degree of coupling in these provinces changed from 0.62 to 0.72 , indicating that the interaction between them is relatively strong, and the relation degree is accordingly tight. As seen in Figure 2, the coupling degrees of 12 provinces in the eastern part of China showed large differences. The maximum was Hebei Province (0.701), the minimum was Guangxi (0.673), and the difference between the maximum and minimum was 0.028 . The coupling degree variations in the nine provinces in the middle part of China were smaller compared with those of the eastern part of China; the maximum was Henan Province (0.691), the minimum was Jiangxi Province (0.653); the difference between them, 0.038 . The coupling degree changes in the 10 provinces in the middle part of China were most pronounced between the eastern and middle part; the maximum was Xinjiang (0.702), the minimum Tibet (0.652), is the difference 0.050 . Combined with the urbanization of each province in 2008 and the moving averages of the adjacent four provinces (Figure 2), we know that the average urbanization values of the three larger belts - namely eastern, central, and western China-are $59.30 \%, 45.63 \%$, and $37.18 \%$, respectively. All three regional belts are in the accelerated development period of urbanization. However, the three belts have large internal differences: The internal differences among the eastern provinces are apparently larger than those in the central and western parts. These regional urban differences may be one of causes of the significant differences in coupling degrees, which we can explain with the "Kuznets curve" of urbanization and farmland variation.

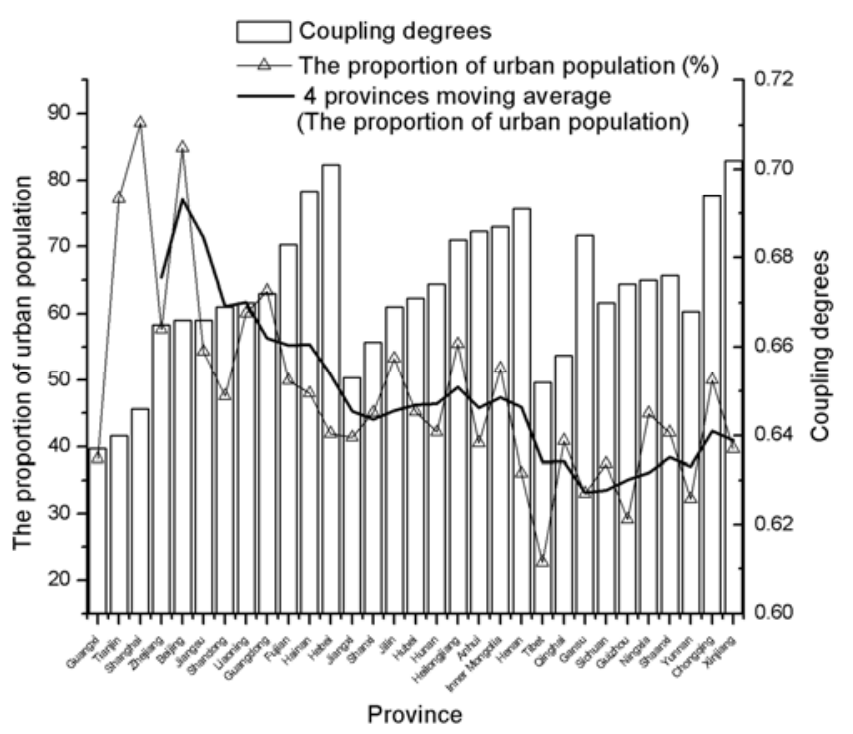

Figure 2. The relationship between the spatial variation of coupling degree and different urban levels.

In the initial phase of urban development, the level of urbanization is relatively low and the speed of urban development is accordingly slow. The urban extension shows epitaxial expansion across the land, leading to the overall decrease of farmland quantity and the decline of farmland quality to some extent, while the regional farmland resources remain within the range of bearing capacity. The interaction impact between urbanization and farmland resources is small, with a low degree of coupling, such as seen in Tibet, Qinghai, Guangxi, and other ethnic minority Autonomous Prefectures. The pace of urbanization is accelerated, however, in the middle period. All of these factors are currently affecting most provinces of China. The conflict between urbanization and farmland resources intensifies, increasing the interaction strength and the coupling degree in the provinces with poor conditions of farmland resources and unreasonable industrial structure. With further development, urbanization enters the later stage. Through adjustments in the structure of industries and effective deployment and intensive use of farmland, the strength of the interaction between urbanization and farmland resources decreases. In addition, the coupling degree also decreases, as seen in areas such as Tianjin, Beijing, Shanghai, and Guangdong. Thus, when urbanization develops to a certain extent, the restraint and limitation of the development of land resources taper off. The interaction between urbanization and farmland resources can be strengthened to some extent through balanced, effective management and control, scientific configuration, and intensive use.

Following is a more detailed description of the spatial distribution patterns and features of coupling between Chinese regional urbanization and ecological environment, involving the dimension of the coupling degree combined with the regional urbanization development index and the urbanization stage division [20]. Overall, the 31 provinces in mainland China 
can be divided into four modes: Low-level Coupled Mode, Antagonism Mode, Transition Mode, and Coordination Mode.

Low-level Coupled Mode. Low-level Coupled Mode encompasses Tibet and Guizhou, two provinces situated in the southwest, accounting for about $6.45 \%$ of the country. Their urbanization level is less than $30 \%$, dominated mainly by secondary and tertiary industry (which together occupy over $80 \%$ of GDP in each province). The GDP per capita is $\$ 2,000$ or less, corresponding with a generally lower level of socioeconomic standards. China has increased its investment in the two provinces year by year, despite the provinces' vast land and sparse population, in accordance with the Western Development Policy. With the rapid development of the economy of the two provinces, the urbanization level has continuously increased and living standards have greatly improved. However, the urbanization level is still in the preliminary stage, with low interaction strength between urbanization and farmland resources. Generally, during this low-level coordination period, the degree of coupling is relatively small (with a value between 0.65 and 0.68 ).

Antagonism Mode. Thirteen provinces (Gansu, Yunnan, Henan, Sichuan, Guangxi, Xinjiang, Anhui, Qinghai, Jiangxi, Hebei, Shaanxi, Hunan, and Ningxia) are included in the Antagonism Mode category. These provinces are located mainly in the middle and western regions, covering about $41.94 \%$ of the nation. The proportion of urban population falls between $30 \%$ and $45 \%$, belonging to the middle stage of urbanization, which primarily focuses on secondary industry (up to $40 \%$ ). GDP per capita is $\$ 2,000$ to $\$ 3,500$. The current accelerated pace of urbanization in this region relies on the urban development of farmland resources. The growing intensity of human development, combined with the constraint, limitations, and negative feedback upon farmland resources, is daily growing more prominent, making these regions hover in the Antagonism Mode category and limiting the interaction between urbanization and farmland resources. The coupling degree ranges from 0.63 to 0.72 . It is worth mentioning that Hebei Province's coupling degree is the highest in this mode (0.701). As the epicenter of regional growth in northern China, Beijing has a "resource deprivation" effect on the surrounding areas, in accordance with the "center-periphery" theory (Fang $\&$ Liu, 2007), and the shorter the distance, the stronger the interaction. Hebei province, as the hinterland of Beijing, is affected by the increased development and industry diffusion of the Beijing-Tianjin-Hebei city cluster. Increases in demand for farmland resources, and the interaction between urbanization and farmland resources, result in a significantly higher coupling degree than those seen in other provinces.

Transition Mode. Transition Mode encompasses 11 provinces: Shanxi, Hubei, Shandong, Hainan, Fujian, Chongqing, Inner Mongolia, Jilin, Jiangsu, Heilongjiang, and Zhejiang, accounting for about $35.48 \%$ of the nation. With a proportion of urban population between $45 \%$ and $60 \%$, these provinces belong to the middle stage of urbanization and are mainly dominated by secondary and tertiary industry (which, except for Hainan province, together account for over $85 \%$ of GDP). The GDP per capita ranges between $\$ 2,500$ and $\$ 6,500$.
The economic development of these provinces has reached a relatively high level. The process of urbanization is in a transition between middle and later periods, and the development of urban-rural integration is becoming apparent. The pressure of spatial urbanization and economic urbanization on farmland resources is relatively heavy in these areas, but because of better and earlier attention to environmental protection in these provinces, their development is within the carrying capacity of farmland resources. The elements of urbanization and farmland resources show a higher level of adaptation and adjustment, reflected in a higher coupling degree (with a value between 0.66 and 0.70 ).

Coordination Mode. The Coordination Mode provinces include Shanghai, Beijing, Tianjin, Guangdong, and Liaoning. These five provinces cover about $16.13 \%$ of the nation, mainly in the east. The proportion of urban population in these provinces exceeds $60 \%$, due to a longer period of urbanization. Urbanization is primarily dominated by secondary and tertiary industry (the total proportion is over 90\%). The GDP per capita is above $\$ 4,500$; thus, socioeconomic levels and living standards are generally high. People in the simultaneous pursuit of material and cultural pursuits pay more attention to ecological and environmental protection. At the same time, due to the high level of science and technology and excellent urban infrastructure, these provinces have the capability to decrease the per capita load of urbanization upon farmland resources via adjustment of industrial structure and intensive land utilization. In general, the interaction between urbanization and farmland resources is in the process of coordination, so that the coupling degree is relatively small (between 0.64 and 0.67 ).

\section{Conclusion}

First, the force of urbanization upon farmland resources is focused on economic urbanization, which comprises a subsystem of association degree, ranging from large to small as follows: economic urbanization $(0.693)>$ social urbanization $(0.681)>$ population urbanization $(0.678)>$ space urbanization (0.655). Therefore, it can be seen that with regard to the constraint of farmland resources upon urbanization, the state constraint of farmland resources is strongest $(0.687)$, farmland resources stress $(0.683)$ is the second strongest, and the social and economic impacts are the smallest (0.663). However, overall, all the factors belong to a relatively strong constraint level.

Second, over time, the entire coupling degree between Chinese urbanization and farmland resources shows a trend of changing from large to small, which indicates that the interactive restrictive function between the two is weakening to some extent. At the same time, however, this progressive decrease has shown fluctuations and internal differences (i.e., eastern region $>$ western region $>$ central region). The reasons underpinning this decrease are the rapid advance of urbanization during the new era and the unbalanced implementation of regional development strategies.

Third, regarding spatial differences, the interaction between 
urbanization and farmland resources is strong in all regions of China. The GRA degree shows a relatively tight correspondence with a high coupling degree, evidence of complexity and particularity. The spatial differences of coupling degree between urbanization and farmland resource are due to internal factors such as regional self-quality, industrial structure, and social development, as well as from external factors like inter-regional differences and regional contact situations. Combined with different development phases of urbanization, the 31 provinces can be divided into four types, consisting of Low-level Coupled Mode, Antagonism Mode, Transition Mode, and Coordination Mode.

Clearly, urban development requires a certain amount of land; however, the conflict between urbanization and farmland resources protection is not absolute. The two-fold solution to the problem is to find a functional relationship of features and rules, suggesting that urbanization development of farmland resources should include the promotion of protection as well. With this win-win strategy, China will finally be able to realize both urban development and protection of farmland resources.

\section{Acknowledgments}

The work described in this paper was supported by the State Key Program of National Natural Science of China (Grant No. 41530745)

\section{References}

[1] Chen, M. X., Lu, D. D., Zhang, H. (2009). Comprehensive evaluation and the driving factors of China's urbanization. Acta Geographica Sinica, 64 (4), 387-398.

[2] Ye, Y. Y., Zhang, H. O., Xu, X. Q. (2011). Study on the relationship between construction land expansion and economic growth pattern in the Pearl River Delta. Geographical Research, 30 (12), 2259-2271.

[3] Zhang, X. X., Zeng, Hui. (2014). Cultivated Land Pressure of China and its Driving Forces Analysis in the Process of Rapid Urbanization Based on Multilevel Panel Model. China Population Resources and Environment, 24 (11), 204-208.

[4] Yu, Q. N. (2001). Land utilization and measures of small town's development in Jiangsu Province. Ecological Economy, $1,29-31$.

[5] Chen, H. J., Deng, L. J., \& Li, H. C. (2010). Research on coordination between the urbanization development and cultivated land change in Chengdu City. Chinese Agricultural Science Bulletin, 26 (1), 312-316.
[6] Tan, M. H., Li, X. B., Lv, C. H. (2004). The expansion of construction land and the occupation to land in lager and medium-sized cities of China in 1990s. SCIENCE IN CHINA Series D: Earth Sciences, 34 (12), 1157-1165.

[7] Zhang, S. Z. (2004). Reflection on the problem of landless peasants in the process of urbanization. Chinese Rural Economy, 2, 44-49.

[8] Li, D., Liu, Y. Z. (2003). Study on the relationship between urbanization development and farmland changes in China. Economic Review, 1, 13-15.

[9] Shi, Y. L, (2000), Analysis of land resources utilization in the process of urbanization in China. China Population, Resources, and Environment, 10 (4), 45-49.

[10] Dai, J. L., Gao, X. L. (2010). Expansion of urban space and land use control in the process of urbanization. Geographical Research, 29 (10), 1822-1831.

[11] Wang, X. M., Wu, Q. Y. (2001). Urbanization construction and intensive utilization of land. China Population, Resources, and Environment, 11 (52), 5-6.

[12] Chen, B. M., Zhang, F. R. (2011). Trend and priority areas in land use research of China. Geographical Research, 30 (1), 19.

[13] Wang, Q., \& Chen, Z. G. (2007). The relationship between urbanization development and farmland changes in China: A survey. Ecological Economics, 2, 135-137.

[14] Cai, Y. Y., Zhang, A. L. (2005). Relationships between cultivated land resource and economic development. China Population, Resources, and Environment, 15 (5), 52-57.

[15] Zhu, H. B., Zhang, A. L. (2006). Analysis on the differences of cultivated land resource quantity security on time and space in China. China Population, Resources, and Environment, 16 (6), 113-117.

[16] Bai, Y., Zhang, Q., \& Shi, Z., \& Zhang, M. (2009) Appraisal of sustainable land utilization based on reusable pressure-stateresponse model for Enping City. Bulletin of Soil and Water Conservation, 19 (4), 209-214.

[17] Liu, Y. B., Li, R. D., Song, X. F. (2005). Grey associative analysis of regional urbanization and eco-environment coupling in China. Acta Geographica Sinica, 60 (2), 237-247.

[18] Fu, L. (1992). Grey System Theory and Application. Beijing: Scientific and Technical Literature Press.

[19] Liu, Y. B., Li, R. D. (2006). Analysis of coupling rule between urbanization and eco-environment in Jiangsu Province. China Population, Resources, and Environment, 16 (1), 47-5

[20] Fang, C. L., Liu, X. L., Lin, X. Q. (2008). Stages correction and regularity analysis of urbanization course of China. Arid Land Geography, 31 (4), 512-523. 